\title{
Study on Physicochemical Properties and Microstructure of Taro Starch in Taizhou
}

\author{
Shuai Shi ${ }^{1}$, Gui-xiang $\mathrm{Qu}^{2}$, Hai-xiang $\mathrm{Xu}^{3}$, Zhi-fang $\mathrm{Li}^{4^{*}}$
}

Jiangsu Agri-Animal Husbandry Vocational College, Taizhou, 225300, China

\begin{abstract}
In order to compare the physical and chemical properties of taro in Taizhou area, taro starch was extracted through the method of spray drying, the blue values, solubility, swelling force, amino acid of amylose and amylopectin and taro starch granules were studied. The results showed that the blue values of amylose were1.16, 0.97, 1.1 in Taixing, jingjiang and xinghua taro respectively, the blue values of amylopectin were 0.19, 0.14, 0.13 in Taixing, jingjiang and xinghua taro respectively. In the comparison of solubility, taixing xianghe taro had a wide distribution, the following sequence was observed: jingjiang xiangsha taro >xinghua longxiang taro. Analyses of amino acids were 3.03mg/100g in taixing xianghe taro starch which was more than the other two taros. The nutritional assessment of amino acids suggested that the taixing xianghe and xinghua longxiang taro contained delicious amino acids which of EAA were 38\% and 44\% respectively. Taixing xianghe taro contained the highest content of phosphorus and potassium, which were $1419 \mathrm{mg} / \mathrm{kg}$ and $8084 \mathrm{mg} / \mathrm{kg}$ separately. Granule morphology showed that starch particles of taixing xianghe taro were smaller than those of taros and the surface was smooth, uniformly. Particle shape was spherical. Xinghua longxiang taro particles with irregular diamond, Jingjiang xiangsha taro starch particles was irregular sphere, and spherical surface was uneven. Taizhou taro was delicate, delicious, which of the taixing xianghe taro was with better quality on the domestic market.
\end{abstract}

Keywords - taro, extraction of starch, quality, analysis.

\section{INTRODUCTION}

Taizhou is acres of water around the city, rich in water sandy loam, especially suitable for the growth of taro. Taixing xianghe taro which is rich in protein, minerals and vitamins, saponins and other ingredients has a unique flavor, beneficial to stomach of high-quality alkaline food. At present, there are few studies on taro in other areas. Jiang Shaotong and Wang Hongpu used to separate and purify polysaccharides from raw taro, studied the chemical characteristics of each component and the effect of immune cells on mice in vitro. Three kinds of polysaccharide fractions were isolated and purified by ion exchange column and gel column ${ }^{[1]}$. Sun Zhongwei studied the extraction of taro starch by water extraction and alcohol precipitation, enzymatic auxiliaries, dialysis, freezing and drying of taro polysaccharides and the nature of taro starch ${ }^{[2]}$. YANG Ying-ying studied the optimized fermentation conditions of purple sweet potato yoghurt, the texture and flavor components were simultaneously studied by using texture technology and headspace solid-phase microextraction and gas chromatography ${ }^{[3]}$, Yu Xin and Xu Wenxing studied the effects of the ratio of raw materials, the content of dough moisture and edible oil on the quality of taro crust in the process of non-fried taro crust, and the study of taro varieties concentrated in Zhejiang Fenghua, Guangxi Lipu and Fujian and other places.

Taizhou taro really took up in people's view in 2012 in the "tongue of China" reported xinghua longxiang taro, so that hundreds of millions of visitors tasted the delicious longxiang taro, but nutrition and starch properties of the Taizhou taro was not reported so far.

In order to effectively play a greater role in the development of taro farmers and the development of taro, it was necessary to study the nutritious composition and related properties of taro in taizhou area. According to the relevant report ${ }^{[2]}$, in order to ensure the quality of starch under water to extract, with ascorbic acid and sodium sulfite to protect color. The basic nutritious contents of Jingjiang xiangsha taro, taixing xianghe and xinghua longxiang were studied. The nutritious composition, solubility, swelling power, transmittance and water absorption of taro starch were studied in order to study the development and utilization of taro in the future.

\section{MATERIALS AND MeTHODS}

\subsection{Materials and Instruments}

Taro: The buds of taixing xianghe taro are red; the leaves of xinghua longxiang taro are dark green, petiole are green and long, leaves and petiole connected with purple halo, the mother taro near spherical, white meat, powder and incense, less 
taro, oval, sticky fleshy; Jingjiang xiangsha taro smooth entrance, sweet, strong sandy, bought in Taizhou every origin; ascorbic acid and n-Butanol purchased from Sinopharm Group (Shanghai) Chemical Reagent Co., Ltd.; sodium sulfite purchased from suzhou china Airlines chemical Technology Co., Ltd.; The above reagents are analytical pure.

DS-1 high-speed tissue crusher were from Shanghai specimen model plant; GYB series of high-pressure homogenizer were from Shanghai Donghua high pressure homogenizer; B-290 small spray dryer were from Beijing Lai Hang Electronics Co., Ltd.; 754 UV spectroscopy Photometer were from Shanghai Jinghua Technology Instrument Co., Ltd.; TD5A-WS-type centrifuge were from Jintan Jinnan Instrument Manufacturing Co., Ltd.

\subsection{Experimental methods}

\subsubsection{The step of taro starch}

marketing taros, which were hypertrophy bulb, upright shape, nattiness and uniform, tissue enrichment, no dry shrinkage and hardening phenomenon, no damage and no metamorphic decay.

The rough surface of taro was removed, washed, cut into thin, and then washed again. The blocks of cut taro were covered with the ratio of material to water 1:2 and $\mathrm{pH} 7$, and then the blocks were placed in a high-speed tissue crusher to a uniform consistency and no lumpy particles. In the same time the particles were ground, $0.01 \%$ ascorbic acid and $0.02 \%$ sodium sulfite $^{[1]}$ solution was added to soak for $70 \mathrm{~min}$, the homogenate extraction was filtrated with 100 mesh sieve, filtrate and filter residue was collected, which was filtered three times with a small amount of water rinse, the filtrate was collected. The filtrate was added to a high pressure homogenizer and homogenized at a pressure of 20-30 MPa. Spray drying, the inlet temperature was $135^{\circ} \mathrm{C}$, the outlet temperature was $75^{\circ} \mathrm{C}$. Exhaust fan $100 \%$, peristaltic pump $20 \%$. The starch was collected after the spray drying and stored in a constant temperature oven $\left(40^{\circ} \mathrm{C}\right)$ and collected.

\subsubsection{Crude separation of amylose and amylopectin}

$5.0 \mathrm{~g}$ sample of taro starch were placed in a $500 \mathrm{~mL}$ beaker, added a small amount of anhydrous ethanol, so that the sample was fully moist, then added $200 \mathrm{~mL} 0.5 \mathrm{~mol} / \mathrm{L} \mathrm{NaOH}$ solutions. The mixing solution was heated in a boiling water bath with constant stirring for $30 \mathrm{~min}$ until complete dispersed. The resulting solution was cooled and centrifuged (4000 r/min, 20 $\mathrm{min}$ ), then the undispersed residue (precipitate fraction) was removed. The centrifuged solution was neutralized with $2 \mathrm{~mol} / \mathrm{L}$ $\mathrm{HCl}$ and added $100 \mathrm{~mL}$ of n-butanol-isoamyl alcohol in a ratio of 3: 1(v/v), and then heated and stirred in the boiling water bath for $20 \mathrm{~min}$. At the same time, the solution was transparent and cooled to room temperature, transferred into $2 \sim 4{ }^{\circ} \mathrm{C}$ refrigerator for $24 \mathrm{~h}$, removed the centrifuge (4000 r/min, $20 \mathrm{~min}$ ), the supernatant was crude amylopectin, the precipitate was crude amylose, the sample were collected for later analysis ${ }^{[5]}$.

\subsection{Purification of amylose and amylopectin}

The precipitate (crude amylose) was transferred to a saturated $\mathrm{n}$-butanol solution $(120 \mathrm{~mL})$. The mixture was then placed in a boiling water bath and stirred until the solution was transparent. The solution was gradually cooled to room temperature and transferred to a refrigerator $\left(2-4{ }^{\circ} \mathrm{C}\right)$ for $24 \mathrm{~h}$, centrifuged $(4000 \mathrm{r} / \mathrm{min}, 20 \mathrm{~min})$, and repeated the above steps 6 times, and then the precipitate was immersed in ethanol for $24 \mathrm{~h}$, washed with anhydrous ethanol several times, And finally the precipitate was dried in a drying oven to obtain a pure amylose, collected for analysis ${ }^{[2]}$. The lower solution was added with $40 \mathrm{~mL}$ of n-butanol-isoamyl alcohol (volume ratio of 1: 1) mixture, heated and stirred in a boiling water bath until the solution was dispersed and transparent, cooled to room temperature, transferred to the refrigerator at $2 \sim 4{ }^{\circ} \mathrm{C}$ for $48 \mathrm{~h}$, centrifuged (4000r/ $\mathrm{min}, 20 \mathrm{~min}$ ), removed the pellet and repeat the above steps 4 times with the supernatant. The resulting supernatant was concentrated under reduced pressure to half of the original volume, added 2 times the volume of ethanol, precipitated, centrifuged, the precipitate was dissolved in $200 \mathrm{~mL} 0.5 \mathrm{~mol} / \mathrm{L}$ hotting $\mathrm{NaOH}$ solution, centrifuged to precipitate, the above precipitated solution was added 2 times volumes of absolute ethanol, dissolved the precipitate in 200 $\mathrm{mL}$ of distilled water, reprecipitated with 2 times of absolute ethanol, washed with anhydrous ethanol several times, finally, precipitated in a drying oven, amylopectin $100 \%$ pure product was obtained collected for analysis ${ }^{[5]}$.

\subsection{Determination of blue value}

Blue value was an indicator of the binding properties of starch and iodine. The absorbance of the formed complex was measured at a wavelength, $0.5 \mathrm{mg}$ of purified amylose and amylopectin was separately added to $50 \mathrm{~mL}$ beaker, added $1 \mathrm{~mL}$ of distilled water and $0.5 \mathrm{~mL}$ of $1 \mathrm{~mol} / \mathrm{L}$ sodium hydroxide solution, then heated in boiling water for $3 \mathrm{~min}$ and then cooled, added $0.5 \mathrm{~mL}$ of $1 \mathrm{~mol} / \mathrm{L}$ hydrochloric acid solution, added $0.1 \mathrm{~g}$ of sodium bitartrate, added water to $45 \mathrm{~mL}$, and added 
$0.5 \mathrm{~mL}$ of iodine solution (mixed solution of potassium iodide and iodine, wherein the content of iodine $0.2 \%$ and the content of potassium iodide $2 \%$ ), the solution was added to $50 \mathrm{~mL}$, mixed, placed at room temperature for 20 min, then measured with a spectrophotometer at $680 \mathrm{~nm}$ wavelength absorbance. With the same concentration of iodine solution as a reference solution. Calculating the blue value according to the following formula: Blue value $=\mathrm{OD}_{680 \mathrm{~nm}} \times 4 /$ the concentration of the sample $(\mathrm{mg} / 100 \mathrm{~mL})^{[5]}$.

\subsection{Determination of Solubility and Swelling Power of Different Taro Starch}

Different starch samples was weighed, added $100 \mathrm{~mL}$ distilled water, heated and stirred at $80^{\circ} \mathrm{C}$ for 30 min, and stirred to make starch solute to reach concentration of $0.4 \%, 0.8 \%, 1.2 \%, 1.6 \%$ and $2 \%$, Centrifuged with speed $3000 \mathrm{r} / \mathrm{min}$ for $30 \mathrm{~min}$. The supernatant was evaporated in a water bath to near drying, and then placed in a constant temperature oven drying, temperature $105^{\circ} \mathrm{C}$ to constant weight. The amount of drying material obtained was $\mathrm{m}$, the solubility was calculated in the following formula ${ }^{[6,7]}$.

$$
\begin{aligned}
& \text { Solubility }=\frac{m}{M} \times 100 \\
& \text { Swelling force }=\frac{W \times 100}{100-S} \times M
\end{aligned}
$$

Formula: $\quad$ m-the supernatant evaporated to dryness weight (unit:g)

M-the weight of starch samples (unit:g)

W-the weight of sediment after centrifugation (unit:g)

\subsection{Scanning electron microscopy}

After the powdery sample was dried, the sample was glued it to holder with conductive glue. The sample holder was placed in the ion sputter and plated a 10 20 nm thick platinum film on the surface. Observation of electron microscopy under different magnification and taking pictures.

\subsection{Determination of drainage rate of different taro starch solution}

Different starch samples was weighed, added $100 \mathrm{~mL}$ of distilled water, heated and stirred at $80^{\circ} \mathrm{C}$ for 30 min, stirred to make starch solution to reach the concentration of $0.4 \%, 0.8 \%, 1.2 \%, 1.6 \%$ and $2 \%$, placed in centrifuge tube, placed in the freezer, removed after $24 \mathrm{~h}$. After its natural thawing, the phenomenon was observed. Then the samples were placed in the freezer refrigerator, frozen repeatedly, centrifuged after drainage, the weight of the precipitate was brought into the equation given below to calculate the starch drainage rate ${ }^{[8]}$.

$$
\text { Drainage rate }=\frac{M-m}{M} \times 100
$$

\subsection{Determination of basic nutritional content in taro}

Phosphorus and potassium elements were determined according to GB/T5009.91-2003 (Determination of minerals in food);

Ash content was determined according to GB 5009.4-2010;

Fat was determined according to GB/T 5009.6-2003 using the first method of soxhlet extraction;

Starch was determined according to GB/T5009.9-2008 using the second method of acid hydrolysis;

Amino acids were determined according to reference GB/T8314-2013 (Determination of total amino acids in food).

The above indicators were measured 3 times, taking the average.

\subsection{Data processing}

Using SAS8.2 software 


\section{RESULTS AND DISCUSSION}

\subsection{Nutritional content of taro}

TABLE 1

THE NUTRITIONAL COMPOSITION OF SOME DIFFERENT TYPE OF TARO $(\mathrm{mg} / \mathrm{kg})$

\begin{tabular}{|c|c|c|c|}
\hline items & taixing xianghe taro & Jingjiang xiangsha taro & xinghua longxiang taro \\
\hline amino acids & $30.86 \pm 0.96$ & $27.2 \pm 0.44$ & $27.28 \pm 0.38$ \\
\hline phosphorus & $1419 \pm 12.34$ & $1263 \pm 13.68$ & $1036 \pm 11.54$ \\
\hline potassium & $8084 \pm 46.54$ & $7621 \pm 49.74$ & $7055 \pm 48.68$ \\
\hline crude fat & $2200 \pm 62.54$ & $2540 \pm 44.58$ & $2580 \pm 52.58$ \\
\hline starch & $91200 \pm 414.56$ & $90240 \pm 420.48$ & $120400 \pm 410.54$ \\
\hline ash & $9240 \pm 64.64$ & $9140 \pm 62.36$ & $9360 \pm 63.42$ \\
\hline
\end{tabular}

Phosphorus was a component of DNA and RNA, which was beneficial to memory and necessary to maintain bones and teeth. We can see from table 1, the content of phosphorus and potassium in Taixing xianghe taro was the highest among the three taro samples, which were $1419 \mathrm{mg} / \mathrm{kg}$ and $8084 \mathrm{mg} / \mathrm{kg}$ respectively. However, the content of potassium in xinghua longxiang taro was the lowest. The highest content of amino acid was $3.086 \mathrm{mg} / 100 \mathrm{~g}$ in Taixing xianghe taro comparable to other two taros. The main component of dry matter was starch, the content was $9 \sim 12 \mathrm{~g} / 100 \mathrm{~g}$, of which starch content in xinghua longxiang taro was the highest $12.04 \mathrm{~g} / 100 \mathrm{~g}$, more suitable as a processing starch varieties. From table 1 comprehensive point of view, the nutritional content of Taixing xianghe taro contrast to other two taros starch can reach the highest value

\subsection{Composition and content of amino acids in taro}

TABLE 2

COMPOSITION AND CONTENT OF AMINO ACIDS OF TARO $(\mathrm{g} / \mathbf{1 0 0 g})$

\begin{tabular}{|c|c|c|c|}
\hline amino acid & taixing xianghe taro & Jingjiang xiangsha taro & xinghua longxiang taro \\
\hline aspartic acid $^{\triangle}$ & $17.10 \pm 1.96$ & $16.90 \pm 1.34$ & $18.63 \pm 1.26$ \\
\hline glutamic acid $^{\triangle}$ & $12.30 \pm 1.34$ & $11.89 \pm 1.28$ & $14.56 \pm 1.42$ \\
\hline alanine $^{\triangle}$ & $4.10 \pm 0.13$ & $4.05 \pm 0.14$ & $5.36 \pm 0.12$ \\
\hline glycine $^{\triangle}$ & $4.51 \pm 0.14$ & $4.32 \pm 0.12$ & $5.45 \pm 0.15$ \\
\hline leucine & $8.62 \pm 0.22$ & $8.42 \pm 0.26$ & $8.86 \pm 0.23$ \\
\hline phenylalanine & $6.82 \pm 0.16$ & $6.76 \pm 0.14$ & $5.92 \pm 0.12$ \\
\hline valine & $5.06 \pm 0.14$ & $5.42 \pm 0.12$ & $5.74 \pm 0.11$ \\
\hline lysine & $5.01 \pm 0.12$ & $5.21 \pm 0.10$ & $6.64 \pm 0.12$ \\
\hline tyrosine & $6.51 \pm 0.20$ & $6.23 \pm 0.15$ & $3.68 \pm 0.05$ \\
\hline isoleucine & $3.65 \pm 0.10$ & $3.42 \pm 0.06$ & $2.68 \pm 0.04$ \\
\hline threonine & $2.62 \pm 0.09$ & $2.44 \pm 0.08$ & $0.76 \pm 0.02$ \\
\hline cysteine & $0.84 \pm 0.02$ & $0.96 \pm 0.04$ & $0.78 \pm 0.03$ \\
\hline
\end{tabular}

Note: $\triangle$ delicious amino acids.

As we can see from table 2, four kinds of umami amino acids and nine kinds of essential amino acids are obtained in taizhou taro after hydrolysis, and the types of amino acid are relatively complete and the proportions are relatively balanced. The proportion of umami amino acids with the total amino acid in taixing xianghe taro and xinghua longxiang taro was $38 \%$ and $44 \%$ respectively, the content of umami amino acids was much higher than that of general food, which may be caused by taizhou taro unique incense and glutinous taste with high nutritional value. In addition, as the main limiting factor of lysine content in three taizhou taro was also rich in content, this can complement the lack of lysine.

\subsection{Determination of blue value in taro starch}

TABLE 3

THE BLUE VALUES OF THE DIFFERENT TYPE OF TARO

\begin{tabular}{|c|c|c|}
\hline taro varieties & amylose & amylopectin \\
\hline taixing xianghe taro & $1.16 \pm 0.12^{\mathrm{a}}$ & $0.19 \pm 0.04^{\mathrm{a}}$ \\
\hline jingjiang xiangsha taro & $0.97 \pm 0.05^{\mathrm{b}}$ & $0.14 \pm 0.06^{\mathrm{a}}$ \\
\hline xinghua longxiang taro & $1.10 \pm 0.08^{\mathrm{a}}$ & $0.13 \pm 0.02^{\mathrm{a}}$ \\
\hline
\end{tabular}

Note: $n=3$; those with the common superscript in the same column mean no significant difference $(P>0.05)$. 
The blue value reflects the ability to bind iodine, and was related to the chain length of amylose or the side chain length of amylopectin. The longer the chain, the higher the blue value. The measured blue values of amylose and amylopectin were different due to their differences in molecular structure and linearity. Because of its high degree of linear polymerization of amylose, the blue value of amylose was generally 0.8 to 1.2 ; because of its short side chain of amylopectin, the general blue value of amylopectin $0.08 \sim 0.22^{[4]}$. The blue values of amylose of taixing xianghe taro, jingjiang xiangsha taro and xinghua longxiang taro were 1.16, 0.97 and 1.10, respectively, and the blue values of amylopectin were $0.19,0.14$ and 0.13 respectively. The blue value of taixing xianghe taro and xinghua longxiang taro was significantly higher than that of jingjiang xiangsha taro $(\mathrm{P}<0.05)$ in amylose, The purity of amylose and amylopectin reached the requirements.

\subsection{Effects of different concentration on solubility and swelling force of taro starch}

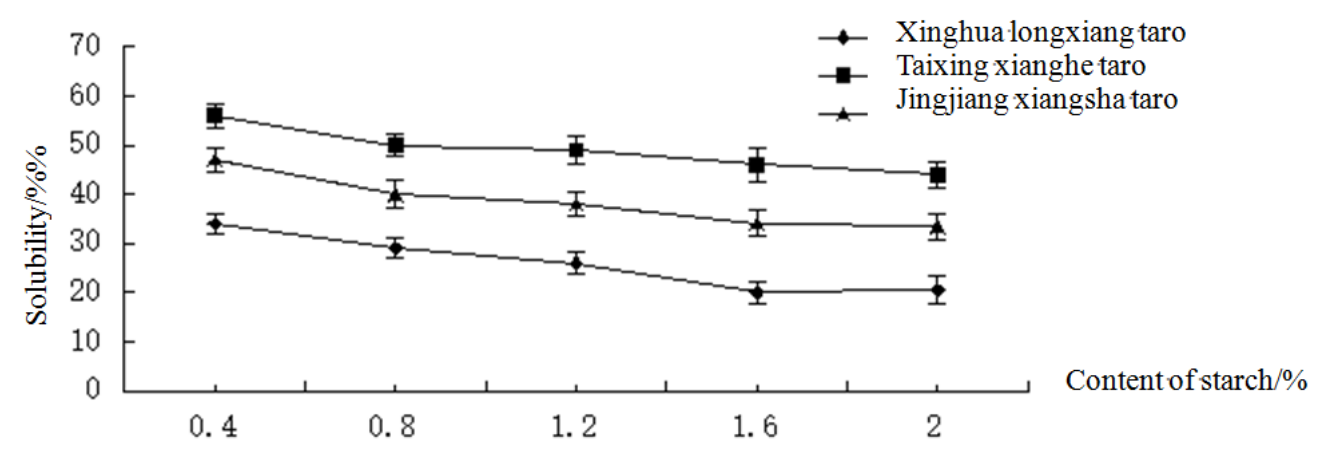

FIG.1 EFFECTS OF CONTENT ON THE SOLUBILITY OF TARO STARCH

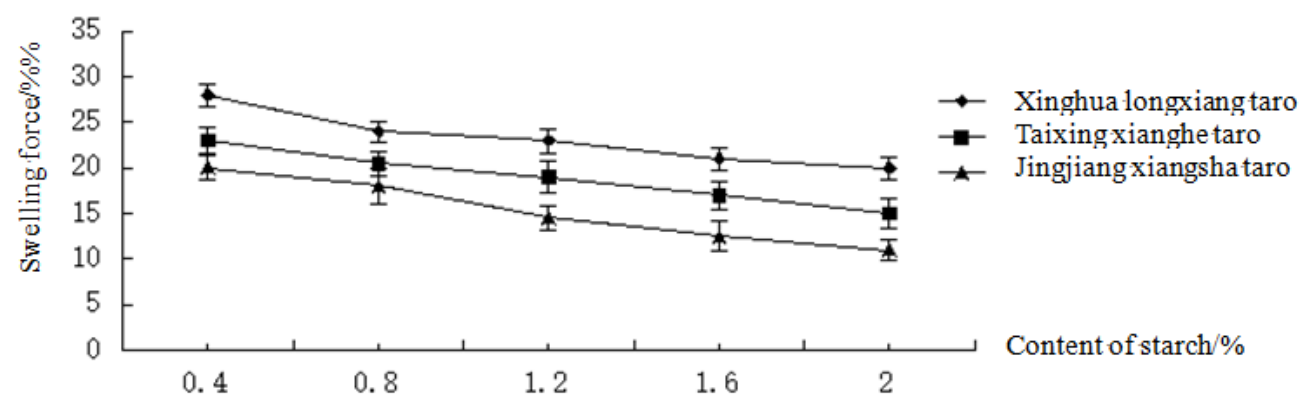

FIG.2: EFFECTS OF CONTENT ON SWELLING POWER OF TARO STARCH

The starch swelling force reflects the characteristics of amylose, and the dissolution of starch was mainly that amylose escaping from the swelling granules, the swelling force indicated the strength of the bonding force inside the starch granules [9]. From Fig. 1 and Fig. 2, we can see that the solubility of taixing xianghe taro was the largest, followed by jingjiang xiangsha and xinghua longxiang taro. As which can be seen from figure 1, with the increasing contention of starch, the corresponding decrease of solubility, swelling force and solubility of starch affect its application in food.

\subsection{Coagulation and sedimentation of taro starch paste}

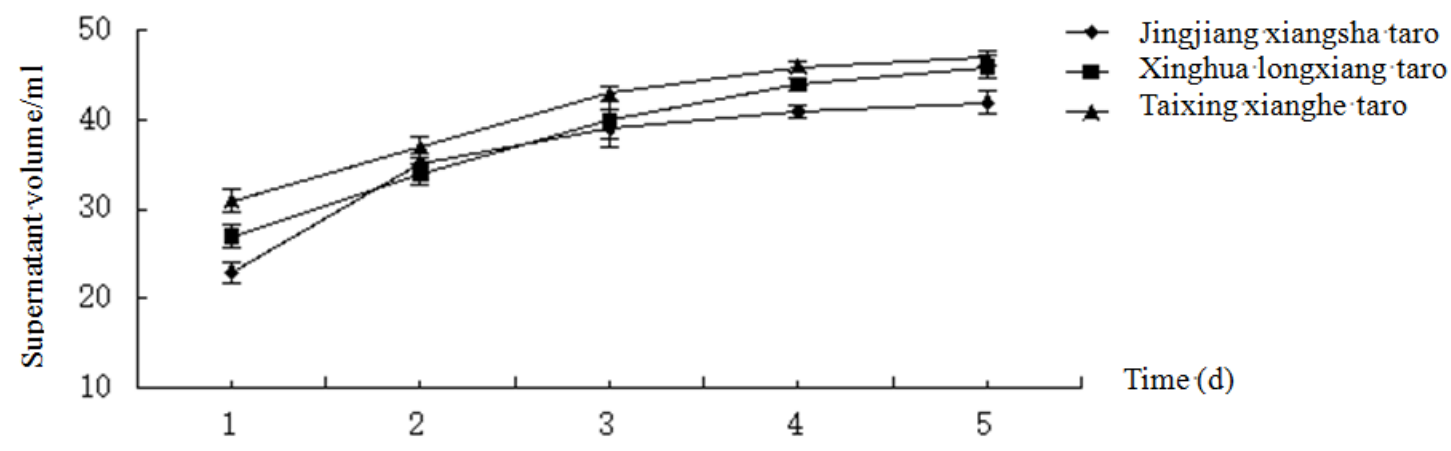

Fig.3: COAgulation AND SEDIMENTATION OF TARO STARCH PASTE 
The speed of coagulation and sedimentation was an indicator of starch properties. As can be seen from Figure 3, in the initial stage, the speed of coagulation and sedimentation was very fast, but after $2 \mathrm{~d}$ the speed became slow. In the order of the speed according to coagulation and sedimentation: taixing xianghe taro>xinghua longxiang taro>jingjiang xiangsha taro. The faster of coagulation and sedimentation in taixing xianghe taro, indicating that the content of starch and the rate of starch extraction were higher.

\subsection{Observations on the morphology of taro granules}

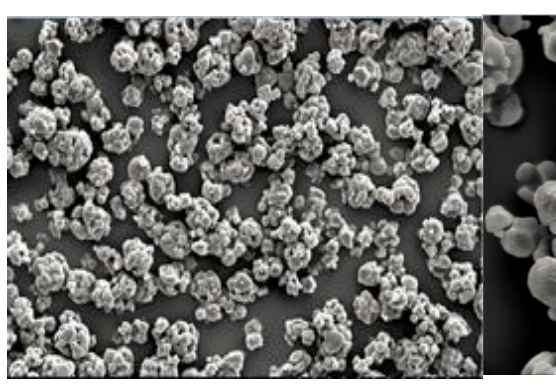

(a) magnifying 800 times

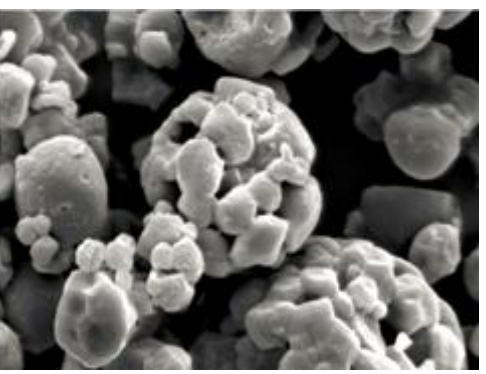

(c) magnifying 4000 times

FIG.4: ELECTRON MICROGRAPHS OF TAIXING TARO STARCH

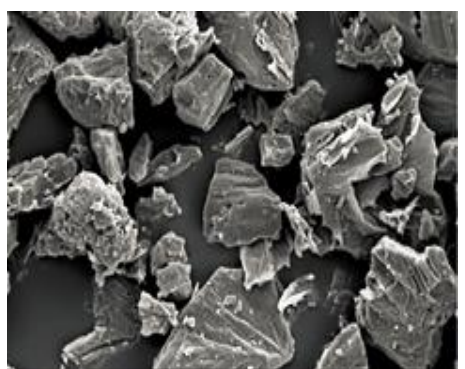

(a) magnifying 800 times

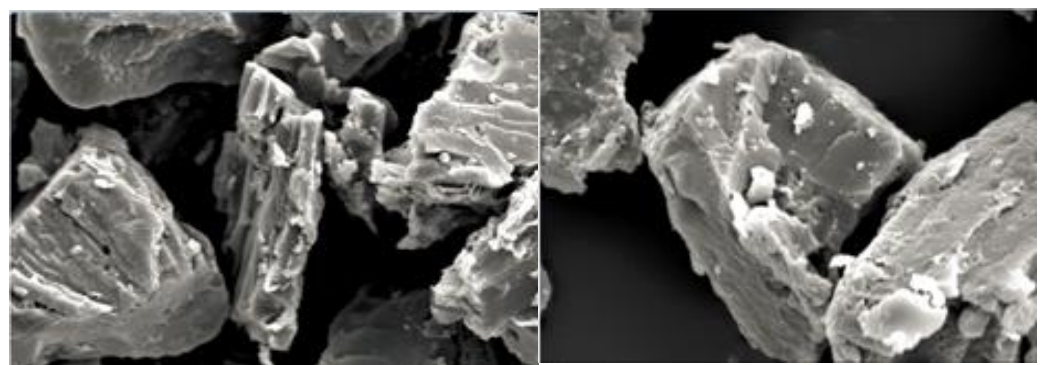

(b) magnifying 2500 times

FIG.5 ELECTRON MICROGRAPHS OF XINGHUA TARO STARCH

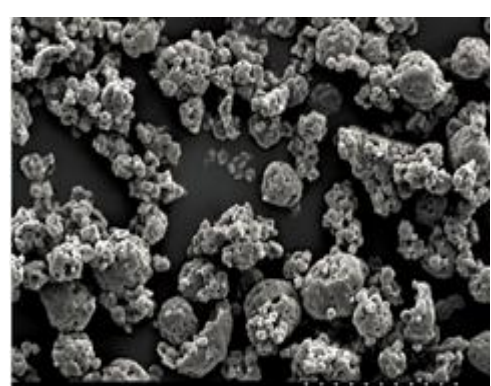

(a) magnifying 800 times

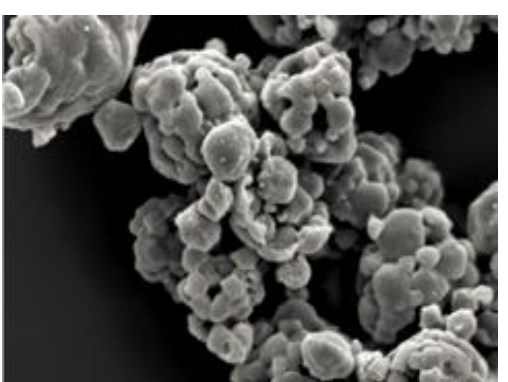

(b) magnifying 2500 times

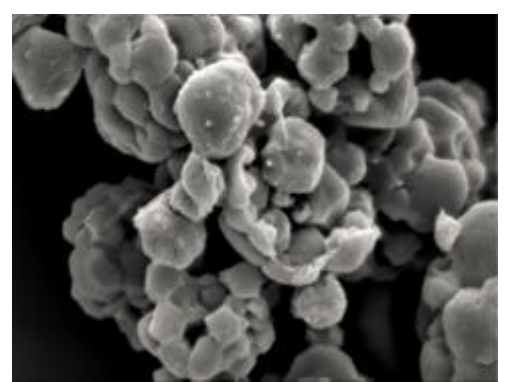

(c) magnifying 4000 times

FIG.6 ELECTRON MICROGRAPHS OF JINGJIANG TARO STARCH

The results of scanning electron microscopy on different taizhou taro were showed from figure 4 figure 6. Starch granules of different taro cultivars have different characteristics in particle size and ultrastructure. From the particle morphology point of view, Taixing taro starch granules smaller and smooth surface, uniform. Particle shape was spherical. Xinghua taro particles were irregular diamond. Jingjiang taro starch granules were irregular spherical, uneven spherical surface. Taro starch granules were small, cooked, the taste smooth, delicate, and hot and cold starch paste stability.

\subsection{Effects of content on drainage rate of taro starch}

As can be seen from figure 7, the drainage rate of jingiang xiangsha taro was highest, followed by xinghua longxiang taro, the curve of drainage rate in jingjiang xiangsha taro almost coincided with that of xinghua longxiang taro, the drainage rate of taixing xianghe taro was smaller than jingjiang xiangsha taro and xinghua longxiang taro.Meanwhile, it can be seen that with the increasing concentration of starch, the drainage rate of starch decreased. It can be inferred by figure 7 that the content of amylose in taizhou taro was as follows: jingjiang xiangsha taro> xinghua longxiang taro> taixing xianghe taro. If the freeze-thawing of starch paste was not stable, which would make the free water drain, thus undermining the original texture of food, so that food quality was declined. 


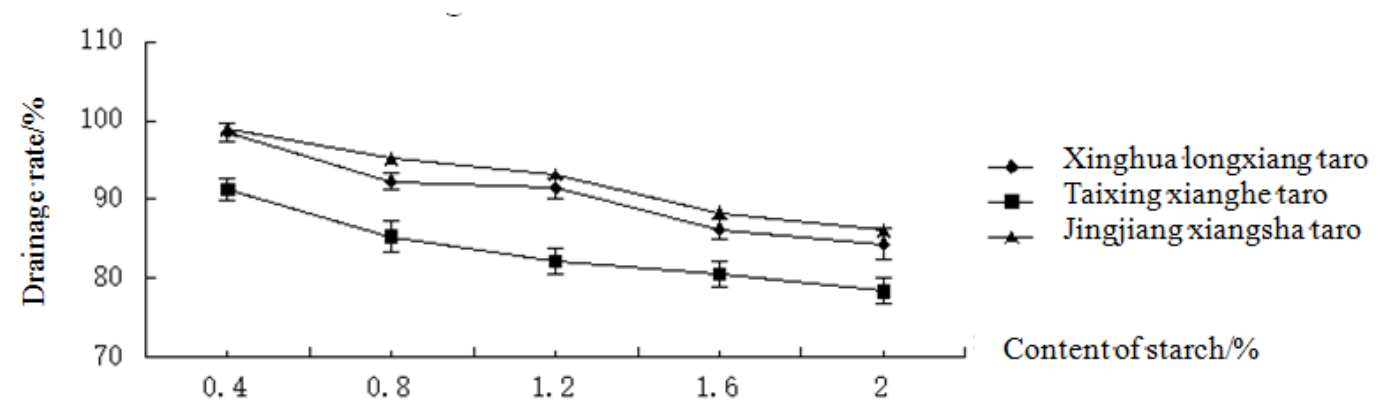

FIG.7 EFFECTS OF CONTENT ON DRAINAGE RATE OF TARO STARCH

\section{CONCLUSION}

The study found that the nutritional ingredients and starch properties on the three kinds of taizhou taro, the study found that the highest total amino acid content of taixing xianghe taro was $3.03 \mathrm{mg} / 100 \mathrm{~g}$; the content of xinghua longxiang taro starch was highest, which was suitable as a food processing starch varieties. The proportion of umami amino acids with the total amino acid in taixing xianghe taro was highest in the comparison of taro varieties, which could be used as raw materials to develop some cooking foods. The starch particles of taixing taro were relatively smaller, which has very good applied prospects on the stability.

\section{ACKNOWLEDGEMENTS}

This work is supported by Fund Project: Agricultural technology support project of Taizhou City, green deep processing technology research and new product development of taro (TN2013001), 2017 College Innovation Venture Project (201712806057Y).

\section{REFERENCES}

[1] Jiang S.T., Wang H.P., Pan L.J. isolation,purification and immunoregulatory property of polysaccharides from taro. Food science, 2013, 34(19):287-292.

[2] Sun Z.W. Studies on the extraction and properties of taro starch[D].Wuxi : Jiangnan university, 2004.

[3] Yang Y.Y. Study on fermentation technology and textural properties and flavor components of purple sweet potato set-style yoghurt [D]. Yantai : Yantai university,2013.

[4] Yun X., Xu W.X., Feng T. Technique's research of backing colocasia esculenta schott chip. Guangzhou food science and technology, 2004, 20(4):64-68.

[5] Wang Yu,Song Wei,Sun Zhong-wei.property investigation for taro starch[J].Journal of the Chinese cereals and oils association, 2006,21(4): 85-90.

[6] Du Xiu-jie. Study on the properties and restraining retrogradation of pinang taro starch [D]. Xiamen : Jimei University, 2012.

[7] Cao X.Z., Liu f., Wu Y.J. Proerties of taro starch. Academic periodical of farm products processing, 2012, (4):57-60.

[8] Yuan J., Chen L., Sun W.J., Study on taro starch extraction and properties of taro starch. Science and technology of food industry, 2012, (19): 252-256. 